\title{
Effects of playing Go for education and psychological treatment
}

\author{
Marc Oliver Rieger ${ }^{\mathrm{a}}{ }^{*}$, Mei Wang ${ }^{\mathrm{b}}$ \\ "University of Trier, Research Cluster "Cultures in Transitions in East Asia and Europe", \\ Universitätsring 15, 54296 Trier, Germany \\ ${ }^{b}$ WHU - Otto Beisheim School of Management, Burgplatz, 2, 56179 Vallendar, Germany
}

\begin{abstract}
The ancient board game Go is traditionally used as educational tool in East Asian countries. Recently, neurobiological and psychological evidence has been found on the effects of Go playing regarding various intellectual and emotional factors. In this survey, we summarize these results and develop a coherent picture of the value that Go can add to education, as well as to psychological treatment. We will also briefly highlight what makes Go special as compared to other commonly played board games.
\end{abstract}

Keywords: strategic board games; Go; weiqi; baduk; brain development; intelligence; emotion; personality; attention deficit hyperactive disorder (ADHD).

\section{Introduction}

Go is likely the oldest board game that is still played today. It originates in China where "weiqi" (Go) was invented around 3000 years ago. In classical East Asian education, it is one of the "Four Arts" (music, Go, calligraphy and painting). Today, it is played worldwide by more than 100 million people (Rieger \& Wang, 2017). While highly popular in East Asia, in Western countries around $1 \%$ of people play Go from time to time (Grootenhuis, 2004).

What is so special about Go as a board game that can explain its traditional role in East Asian education? After all, the benefits of games, e.g., for education are wellknown (Boghian et al., 2019). Why would one expect to see other effects of Go on the human brain than, let's say, for chess, which is a comparable game which has already been widely studied in psychology?

Possible reasons can be found in certain structural features of the game:

1. Need for intuitive play

In many games, the number of possible moves in every situation is rather limited. In chess, e.g., at the start of the game each player has 20 different moves. If this number is rather small, it is possible to consider each move and to access its consequences (at least briefly). In Go, the typical number of moves is between 100 and 300. Indeed, the total number of different positions in Go is at least 10120 times larger than the number of positions in chess (Tromp, 2016; Steinberger, 2015). With so many possibilities, brute force computations and learning by heart become less meaningful and are frequently replaced with more abstract concepts and intuitive heuristics in evaluating moves and positions.

2. Symmetry of the game

Unlike, e.g., chess, Go is completely symmetric: the game would not change when turning the board by 90 degree or mirroring the position. This implies that

* Corresponding author.

E-mail address: mrieger@uni-trier.de; mei.wang@whu.edu

DOI: 10.51865/JESP.2021.2.11 
a player will need to develop his geometric orientation skills (turning positions around or mirroring them in his mind) in order to recognize previously encountered patterns.

\section{Non-locality of the game}

Go is played on a rather large board $(19 \times 19)$ and a move on one end of the board can affect indirectly a position elsewhere. It is typical for a game to jump back and forth between very different areas of the board. This inter-connectivity between local situations is another typical feature of Go. It allows for creative solutions to problems somewhere on the board by exploring a situation elsewhere. Easy proxies for the status of a game (like in chess where counting the remaining figures of the players weighted by their strength is already a very good indicator) are in Go entirely missing (Crasmaru, 1998), thus in Go, different from most other games, a "holistic" thinking style is required.

The difference between Go and other board games like chess or checkers can also be seen in computer play: in most board games, computer programs could defeat the best humans since the 1990s by using fairly simple brute force computations with an evaluation function. In Go, however, it took 20 more years and an enormous research effort in artificial intelligence by Google scientists to defeat a world champion in Go (Silver et al. 2016).

In this article, we review scientific studies on neurobiological, psychological, educational, and health effects of Go playing, mainly for children and young adults.

\section{Effects on brain development and functioning}

\subsection{Neurobiological results}

In recent years, the effect of playing Go has been measured directly on the neurobiological level, using fMRI (functional magnetic resonance imaging) or PET (Positron emission tomography). Such studies can show objectively differences between brain structures and brain functioning with the caveat that it is not always easy to translate findings into behavioral differences, and that a causality is difficult to establish.

Chen et al. (2003) were comparing subjects playing Go with subjects playing chess regarding their brain activity using fMRI. They found increased activity in areas in the mid-dorsal, dorsal prefrontal and other brain regions that are "generally engaged in attention, spatial perception, imagery, manipulation and storage in working memory, retrieval in episodic memory, and problem solving." Different from chess, they noticed an increased activation of the right hemisphere which could be a sign of a higher degree of intuition and creativity needed in Go.

Ouchi, et al. (2005) studied the difference between professional and amateur Go players while thinking about opening and "life and death" problems using PET. In amateur players more brain regions were active, while in professional players specialized areas were active, in particular, areas for imagery and motor imagination.

Jung et al. (2013) investigated the brain structure and functional connectivity (FC) of Go players using voxel-based and FC methods. They found that, compared to novices, Go experts had a higher degree of grey matter in the nucleus accumbens (part of the mesolymbic system that produces feelings of joy) and a lower amount of grey matter in the amygdala (connected to emotional decisions). They also found a higher degree of functional connectivity between amygdala and the medial prefrontal cortex (cognitive decision making) and a lower connectivity between the nucleus accumbens and the medial prefrontal cortex. This suggests that playing Go has an effect on the overall structure of the brain that seems to reduce emotional decisions, but at the same time strengthening the connections between emotional and cognitive decision making.

In a similar spirit, Lee, et al. (2010) study the difference in white matter of various brain regions between Go players and a control group. Go players developed larger regions of white matter in the frontal, cingulum and striato-thalmic areas. These areas 
are related to attentional control, working memory, executive regulation, and problemsolving.

Taking the results of these studies together, Go seems to improve the ability of rational decision making, attention, imagery, motor imagination and working memory. While these are structural results, we will find behavioral evidence for these effects in some of the studies discussed below.

\subsection{Empirical evidence}

Measuring long-term effects of playing Go on cognitive abilities is inherently difficult, since no long-term longitudinal data exists. Rieger \& Wang (2017) chose a different approach: in a large-scale survey $(\mathrm{N}=327)$ among Go players attending amateur tournaments they find statistically significant correlations between playing strength and intelligence (as proxied by the cognitive reflection (CRT), see Frederick (2005)), and between playing frequency and patience and theory of mind (measured as in Nagel (1995) and Selten \& Nagel (1998)).

\section{Average CRT score}

a. Go players at tournaments

b. MIT students

c. Finance professionals

b. Princeton students

b. US students

d. Malaysian youth (21-30 years)

e. Mixed Brasilian subjects

f. Broad sample of US citizens

b. University of Toledo students

g. Sri Lanka, SME owners

g. Sri Lanka, wage workers

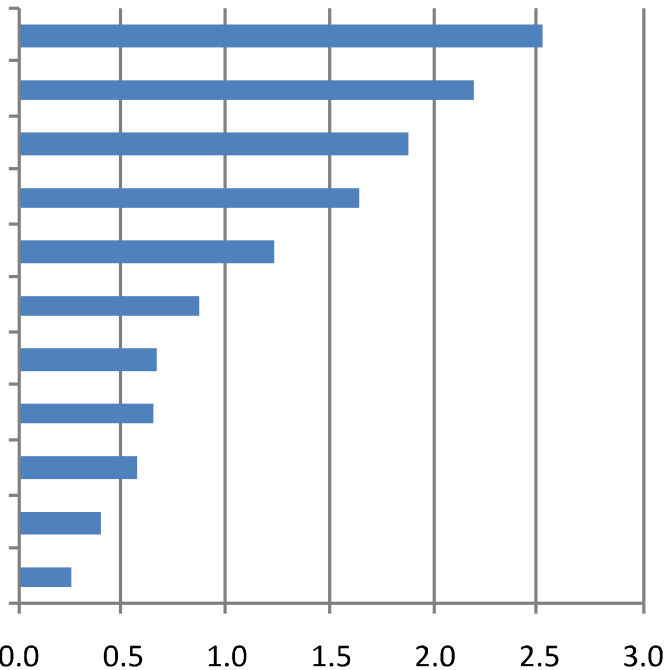

Fig. 1. Cognitive reflection scores (CRT) of subject groups from selected studies in comparison with the level measured for Go players (taken from Rieger \& Wang (2017), references to the various studies are provided there).

The CRT score among Go players exceeded by far average population values and other previous measurements, including data collected among financial professionals, and students at top universities (Fig. 1).2 The positive effect of Go playing frequency on theory of mind might be a training effect: in order to anticipate an opponent's plans, a player needs to think from his or her point of view.

\section{Effects on children and adolescents}

\subsection{Effects on intelligence}

Intelligence has many facets. Some aspects of the effects of Go playing have been studied in details. For example, based on the above-mentioned symmetry properties of Go, researchers suspected that studying Go may improve certain spatial transformation and motor imaginary tasks. This has been backed up by neurobiological studies in

${ }^{2}$ One cannot exclude, however, that this is due to self-selection: maybe only intelligent people find Go attractive (see Kholiavko et al. (2020) for more on selection processes for extracurricular activities). 
proficient Go players, see Section 2, particularly Ouchi et al. (2005), but also by field studies: in a quasi-experimental setting with $\mathrm{N}=103$ children of two preschools in Taipei, half of them entered a Go program twice a week for six weeks. Subsequently, these children improved in spatial transformation tasks substantially (Xiao, 2014). A similar study with $\mathrm{N}=60$ kindergarten children where half of them took a 12 weeks program of Go showed a significant increase in figure reasoning ability in a standard intelligence test (Ke, 2013).

Another facet of intelligence that is often connected with Go is logical reasoning. We have seen already that neurobiology studies suggest that parts of the brain that are in charge of cognitive decision making increase connectivity in long-term Go players (see Section 2). Studies on children support that: twenty junior high school students of the 7th grade that had no previous knowledge on Go, attended a weekly two hours class for 8 weeks, followed by two weeks of a Go tournament. A standard intelligence test ("Raven's Standard Progressive Matrices Plus") was administered before and after the intervention. The test results improved significantly by the intervention $(\mathrm{Li}, 2013)$.

The aforementioned studies were all conducted in East Asia, and we might wonder whether they can be carried over to Western cultures where expectations and support by the parents as well as the students' attitudes towards learning will differ. We therefore report some data that have been recorded at a high school in Berlin, Germany, but not been analyzed previously. $\mathrm{N}=24$ children at that school started at different school years to participate at a Go class. Their grades in mathematics have been recorded for the three semesters before and after entering the Go class. When comparing their grade averages before and after starting with Go, a significant improvement (Wilcoxon signed-rank test: $\mathrm{p}=0.028$ ) was noted. ${ }^{3}$ The distribution of grade changes can be seen in Fig. 2: half of the children improved their math grades, although studying Go obviously took additional time that they could not spend for studying mathematics. Note that these children were in different classes and that grades averages among all school children were basically the same in all of these years.

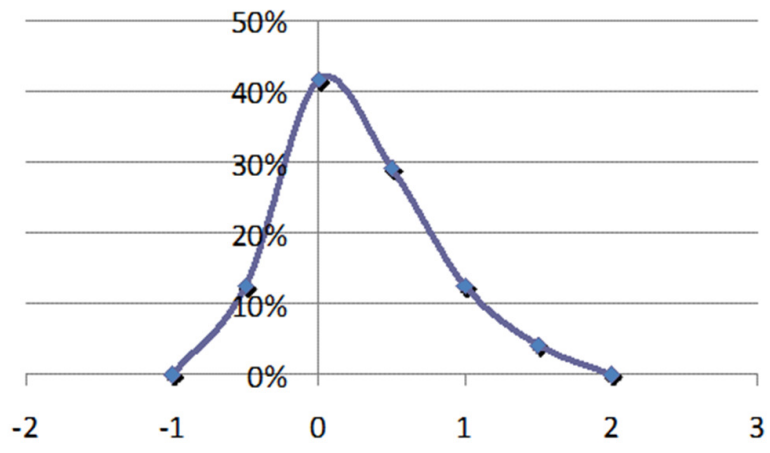

Fig. 1. Distribution of changes in mathematics grades among high school students who started to learn Go. Positive changes correspond to improved grades. Grades were given on a scale from A to $\mathrm{F}$ (1 to 5).

In conclusion, studying Go helped the children to improve their math grades. The precise mechanism for that cannot be deduced from this data, but an improvement in logical reasoning, cognitive decision making or attention are possible explanations.

Given the significant changes in some categories for older preschool children (Ke, 2013; Xiao, 2014), but the much stronger effect for junior high school students in the above mentioned German high school data and in $\mathrm{Li}$ (2013), one might wonder whether Go is indeed more helpful for older children. In Western countries the typical age for children to learn Go is substantially older than in East Asia where children usually start

\footnotetext{
${ }^{3}$ The effect was similar when only considering the grades of one or two semesters before and after.
} 
learning Go in kindergarten, thus beneficial effects might be larger in Western countries.

In conclusion, there is ample evidence that Go, particularly for older children, improves cognitive abilities. The effect does not seem to be restricted to East Asian children and we agree with the conclusion from the survey article by Cai \& Huang (2013) on the effects of Go on higher level thinking in children: Go indeed "enhances cognitive thinking and other comprehensive abilities".

\subsection{Effects on creativity}

While positive effects on certain facets of intelligence can be attributed also to playing other board games, there is a particularly strong emphasis in Go on intuition and creativity, as has been pointed out in Section 1.

We had mentioned in Section 2 the study by Chen et al. (2003) that provides a neurobiological basis for a connection between Go playing and creativity. There is, however, also some empirical evidence for a causal effect in the study by Li (2013): a standard psychological test on creativity, the Creativity Assessment Packet (CAP), was used for high school students who took part in a Go teaching program. A significant improvement was noticed in the measurements, in particular for fluency, openness and originality.

There is unfortunately still a comparative lack of studies on the effects on creativity, but the above-mentioned results at least suggest a positive impact of Go on creativity.

\subsection{Effects on emotion and personality}

East Asian Parents expect Go to help their children to develop concentration and emotional stability (Kim, 2005). "Even parents who do not know Go are aware that it is not bad for a child to learn Go" (Kim \& Lee, 2004). This belief motivated a number of studies that investigated the impact of Go on emotion and personality, in particular on attention, extraversion, neuroticism, psychoticism and volition.

Probably the largest and best controlled psychological study on this subject is the study by Xu \& Kong (2009) and Xu (2009). In total $\mathrm{N}=852$ children were randomly assigned to a Go learning group and a control group. Tests reveiled a positive effect of Go on attention and willpower. Differences were particularly strong in attention, persistence, decisiveness, and confidence. It was also observed that these differences were the same for boys and girls, underlining the fact that Go is not a "boy's thing".

Similar results have been found by Huang, Kong \& Hu (2006) who used different psychological tests for children of grade 4-6 in two elementary schools in China, one focusing on Go. Children in the Go school had better results in extraversion, neuroticism and psychoticism. Their attention was higher and they showed a higher volition.

In conclusion, there seem to be robust evidence for a positive effect of studying Go on emotions and personality of children.

\subsection{Attention deficit hyperactive disorder (ADHD)}

The effect of Go on attention and, more generally, on personality provides a motivation for application on the treatment of attention deficit hyperactive disorder (ADHD). Kim et al. (2014) studied N=34 children (7-12 years old), half of which were diagnosed with ADHD, but have not been treated with drugs, and half of which were a control group, matched for age and gender. After 16 weeks of intense Go training (2 hours every day, from Monday to Friday), significant improvements in the ADHD rating scale, inattentive score and short-term memory (digit total score) were found. 
Moreover, EEG measurements of relevant brain functions of the ADHD children improved significantly as well.

The authors conclude that studying Go seems to be "effective for children with ADHD by activating hypoarousal prefrontal function and enhancing executive functions" in the brain.

\section{Summary}

We have seen in this survey article that studying and playing Go has various effects that are important for education and health. Studies in both East Asia and the West, suggest that it can improve certain facets of intelligence, attention, and important personality aspects. It has also been connected to an improvement in creativity and emotional intelligence. Moreover, Go might also be useful for psychological treatment, in particular in ADHD.

\section{References}

1. Boghian, I., Cojocariu, V. M., Popescu, C. V., \& Mâţă, L. (2019). Game-based learning. Using board games in adult education. Journal of Educational Sciences \& Psychology, 9(1).

2. Cai, G. \& Huang, X. (2013), 'Discussion on the effect of go training on children's high-level thinking', Naping Special Education, 37-47.

3. Chen, X., Zhang, D., Zhang, X., Li, Z., Meng, X., He, S. \& Hu, X. (2003), 'A functional MRI study of high-level cognition ii. the game of GO', Cognitive Brain Research 16, 32-37.

4. Crasmaru, M. (1998), On the complexity of tsume-go, in 'International Conference on Computers and Games', Vol. 1558, Springer, pp. 222-231.

5. Frederick, S. (2005), 'Cognitive reflection and decision making', Journal of Economic Perspectives 19(4), 25-42.

6. Grootenhuis, R. (2004), 'Bekanntheitsanalyse Go 2004' [Awareness Analysis of Go], Deutsche Go Zeitung 79(4), 38-39.

7. Huang, H., Kong, K. \& Hu, Y. (2006), 'The effect research of pupil's personality development by go games', Psychology development and education 2, 12-17.

8. Jung, W. H., Kim, S. N., Lee, T. Y., Jang, J. H., Chi-Hoon Choi and, D.-H. K. \& Kwon, J. S. (2013), 'Exploring the brains of Baduk (Go) experts: gray matter morphometry, resting-state functional connectivity, and graph theoretical analysis', Frontier in Human Neuroscience 7(Article 633), 1-17.

9. Ke, Y. (2013), The impacts of go learning on children's figure reasoning ability, Master's thesis, National Pingtung University of Education.

10. Kholiavko, N., Detsiuk, T., \& Tarasenko, O. (2020). Extracurricular activity of engineering students: trends and motives. Journal of Educational Sciences \& Psychology, 10(1).

11. Kim, B. (2005), 'A study on parents' consciousness on children's baduk education', Baduk Studies 2, 38-72.

12. Kim, B. \& Lee, J. (2004), 'A study on the motivation for participation in special ability education in go', Proceedings of the 2nd Go conference pp. 79-91.

13. Lee, B., Park, J.-Y., Jung, W. H., Kim, H. S., Oh, J. S., Choi, C.-H., Jang, J. H., Kang, D.-H. \& Kwon, J. S. (2010), 'White matter neuroplastic changes in long-term trained players of the game of "baduk" (GO): A voxel-based diffusion-tensor imaging study', NeuroImage 52, 9-19.

14. Li, Y. (2013), A study of using go teaching to promote junior high school students logic reasoning and creative thinking, Master's thesis, Tamkang University, Taiwan.

15. Nagel, R. (1995), 'Unraveling in guessing games: An experimental study', American Economic Review 85(3), 1313-1326.

16. Ouchi, Y., Kanno, T., Yoshikawa, E., Futatsubashi, M., Okada, H., Torizuka, T. \& Kaneko, M. (2005), 'Neural substrates in judgment process while playing go: a comparison of amateurs with professionals', Cognitive Brain Research 23, 164-170.

17. Rieger, M. O. \& Wang, M. (2017), 'Cognitive skills and competence in go', SSRN working paper. 
18. Selten, R. \& Nagel, R. (1998), 'Das Zahlenwahlspiel - Ergebnisse und Hintergrund [the guessing game - results and background]', Spektrum der Wissenschaft, pp. 16-22.

19. Silver, D., Huang, A., Maddison, C. J., Guez, A., Sifre, L., van den Driessche, G., Nham, J., Kalchbrenner, N., Sutskever, I., Lillicrap, T., Leach, M., Kavukcuoglu, K., Graepel, T. \& Hassabis, D. (2016), 'Mastering the game of go with deep neural networks and tree search', Nature 529, 484-489.

20. Steinerberger, S. (2015), 'On the number of positions in chess without promotion', International Journal of Game Theory 44, 761.

21. Tromp, J. (2016), The number of legal go positions, in v. d. H. J. Plaat A., Kosters W., ed., 'Computers and Games', Vol. 10068, Springer, Cham.

22. Xiao, A. (2014), The effect of go game on children's spatial capacity and attention, Master's thesis, National Taiwan Normal University.

23. Xu, P. (2009), 'Impacts of game of Go on attention of children', Psychological Science 2, 364 367.

24. Xu, P. \& Kong, K. (2009), 'Impact of game of go to the willpower of children', Psychological Research 2, 64-68. 